\title{
Anchoring lumen-apposing metal stent with coaxial plastic stent for endoscopic ultrasound-guided drainage of pancreatic fluid collections: any benefit?
}

\author{
Saad Emhmed Alia , Karim Benrajab ${ }^{b}$, Houssam Mardini ${ }^{b}$, Leon Suc, Moamen Gabrb, Wesam M. Frandah ${ }^{\text {b }}$ \\ University of Kentucky Medical Center, University of Kentucky, USA
}

\section{Abstract}

\section{Introduction}

Acute pancreatitis is one of the top 3 gastrointestinal disorders requiring hospital admission in the USA [1,2]. It is

\footnotetext{
Department of aInternal Medicine, Division of Hospital Medicine (Saad Emhmed Ali); bInternal Medicine, Gastroenterology and Hepatology (Karim Benrajab, Houssam Mardini, Moamen Gabr, Wesam M. Frandah); cStatistics, College of Arts and Sciences, College of Public Health (Leon Su), University of Kentucky, USA

Conflict of Interest: None

Correspondence to: Saad Emhmed Ali, MD, 800 Rose St, Lexington, KY 40536, USA, e-mail: saad.ali@uky.edu This study was submitted to the DDW 2019, San Diego, California, USA
}

Received 16 June 2019; accepted 21 July 2019; published online 23 September 2019

DOI: https://doi.org/10.20524/aog.2019.0414 a self-limited disease, but can be fatal in patients with organ failure if it persists for more than 2 days [3]. Local complications of acute pancreatitis have been classified according to the revised 2012 Atlanta international consensus [4]. Postpancreatitis fluid collections (PFCs) that persist for more than 4 weeks and have a well-defined wall are divided into pancreatic pseudocyst (PP), which contains homogenous fluid, and walled-off pancreatic necrosis (WOPN), which is heterogeneous and contains liquid and solid material [4]. Until lately, these collections were drained by needle-knifing into the cavity, without or with endoscopic ultrasound (EUS) guidance, and placing multiple double-pigtail stents (DPSs) [5,6]. Even though the DPSs aid the drainage of liquid material, they are not effective in draining solid material from the cyst cavity and several exchanges are required over guidewires and serial balloon dilatations. In addition, the procedure is timeconsuming and relatively challenging. With the introduction of EUS-guided lumen-apposing metal stents (LAMSs) in 2012 (Axios, originally Xlumena Inc, Mountain View, CA; now 
Boston Scientific, Marlborough, MA), draining PFCs have become much more accessible. Given its large diameter (up to $15 \mathrm{~mm}$ ), a standard or a therapeutic gastroscope can be passed through the stent into the cavity, allowing necrotic tissue debridement in the setting of WOPN [7,8]. Many centers, including our own, have been placing LAMSs for drainage of PFCs because of their ease of deployment. Although several studies have been published on the use of LAMSs for PFCs, there are limited data on the safety and efficacy of placing coaxial DPSs within LAMSs for draining PFCs [9-12]. Currently, there are no predefined criteria for patient selection as regards using the LAMSs alone or LAMSs plus DPSs during the drainage of PFCs. We sought to compare the outcomes of patients who received LAMSs alone and those who received both LAMSs and anchoring DPSs for EUS-guided drainage of PFCs.

\section{Patients and methods}

This was a retrospective cohort study conducted at a tertiary healthcare center from January 2016 until March 2018. Included were patients with symptomatic PFCs who underwent EUS-guided transmural drainage using LAMSs, with or without DPSs. The 2 approaches (LAMS vs. LAMS plus DPS) were compared. The database was retrospectively reviewed to evaluate the safety and efficacy of using the LAMSs with or without DPSs for draining PFCs, after approval had been obtained from the local institute's Internal Review Board. Patient's imaging data, including computed tomography or magnetic resonance imaging, were reviewed along with the EUS findings. PFCs were classified into PP and WOPN according to the revised 2012 Atlanta international consensus [4]. Inclusion criteria were age above 18 years, provision of informed written consent, symptomatic PFCs requiring transmural drainage, as specified by the Working Group of the International Association of Pancreatology [13], and PFCs that persisted for more than 4 weeks with a mature cavity wall on imaging. Exclusion criteria were any other type of fluid collection (fluid was sent for analysis of amylase level, cytology, and carcinoembryonic antigen level during the EUS), immature cavity wall, drainage with stents other than LAMSs, pregnancy, lack of patient stability for endoscopy, and severe coagulopathy or thrombocytopenia.
All EUS-guided drainage procedures of WOPN and PP were performed by 2 interventional endoscopists. The decision to deploy LAMSs alone or with DPSs was at the discretion of the endoscopist. All drainage procedures were performed under general anesthesia with orotracheal intubation. PFCs were evaluated and drained using a linear echo-endoscope (180T; Olympus America, Center Valley, Pa). LAMSs were placed in all patients (AXIOS or Hot AXIOS; 10 or $15 \mathrm{~mm} \times 10 \mathrm{~mm}$; Boston Scientific, Marlborough, Massachusetts, USA) (Fig. 1 A,B,C). Depending on the endoscopist's decision, DPSs $(10 \mathrm{Fr} \times 5 \mathrm{~cm}$; Cook Medical, Bloomington, Indiana, USA) were placed coaxially through the LAMSs (Fig. 2). All patients received intravenous broad-spectrum antibiotics before the procedure. Follow-up imaging studies were performed every 3 weeks. Once the PFCs were entirely resolved, the stents were removed by a repeat endoscopy using grasping forceps (Boston Scientific).

The primary endpoint of this study was to evaluate the efficacy (PFCs resolution) and safety (adverse events) of LAMSs, with or without DPSs, used to drain WOPN and PP. The secondary endpoint was the technical success in placing LAMSs and DPSs. We defined and graded the adverse events according to the American Society for Gastrointestinal Endoscopy's Lexicon [14]. The timing of adverse events was classified as early if it happened during or immediately after the procedure, post-procedure up to 14 days after, and late after 14 days. We defined technical success as the ability to place the stents successfully, and clinical success as the resolution of PFCs on cross-sectional imaging to $<2 \mathrm{~cm}$ with no further interventions needed, together with an improvement in the patient's symptoms.

\section{Statistical analysis}

Categorical data were analyzed using chi-square tests, and continuous variables were analyzed using 2-sample t-tests. A statistical significance level of 0.05 was used, and all analyses were completed in SAS 9.4 (SAS Institute Inc., Cary, NC, USA).

\section{Results}

Fifty-seven patients with PFCs (23 female and 34 male; average age 47 years) were treated by 2 advanced endoscopists
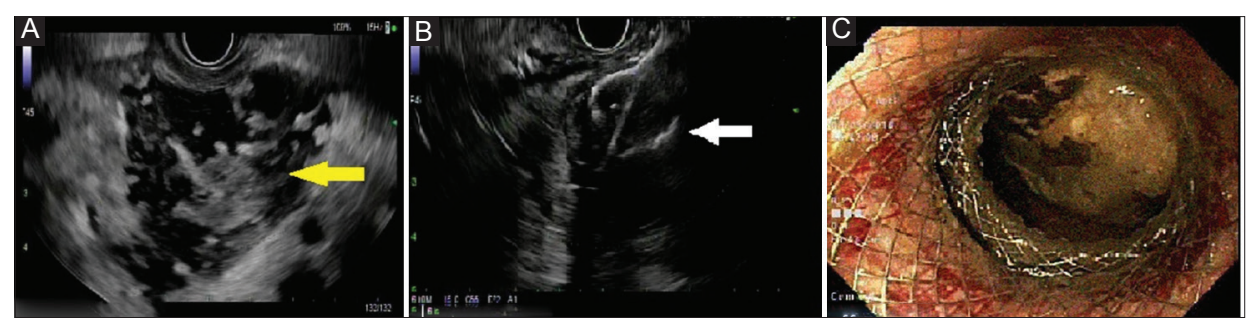

Figure 1 (A) Endoscopic ultrasonographic image showing a large walled-off pancreatic necrosis with the yellow arrow pointing to a solid material in the collection. (B) Endoscopic ultrasonographic image showing the deployment of lumen-apposing metal stent (LAMS) with the white arrow pointing to the proximal flange of the LAMS. (C) Endoscopic image showing the LAMS after deployment with a large amount of necrosis in the cavity 


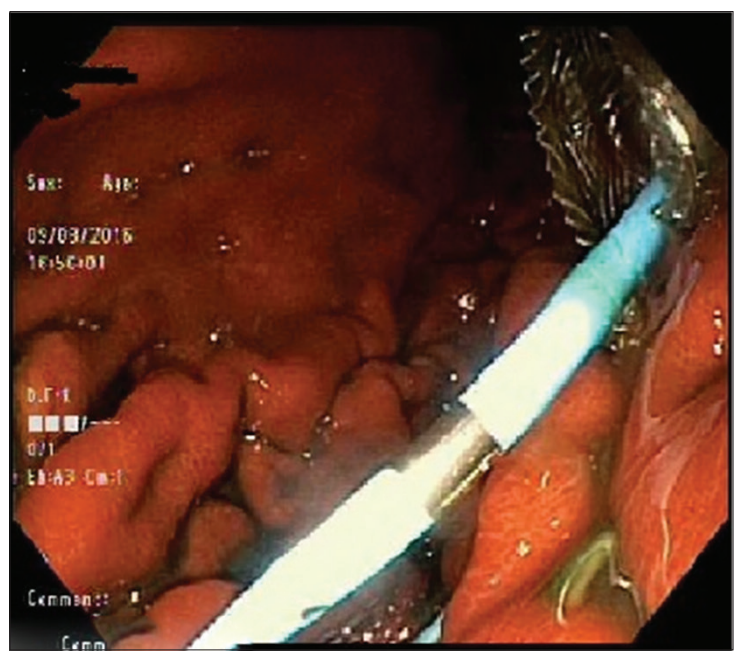

Figure 2 Endoscopic image showing the lumen-opposing metal stent with double-pigtail plastic stent over 26 months. Twenty-one (37\%) patients received LAMSs alone, while $36(63 \%)$ patients received LAMSs plus DPSs. Forty-three patients had WOPN, and 14 patients had PP. We classified the size of the PFCs into 3 groups: group 1 (0-50 mm), group 2 (51-100 $\mathrm{mm})$, and group 3 (>100 $\mathrm{mm})$. The average PFCs size in the LAMSs and LAMSs plus DPSs groups were $90 \mathrm{~mm}$ and $100.5 \mathrm{~mm}$, respectively (Table 1 ).

The technical success rate in placing LAMS and DPS was $100 \%$. The drainage site was transgastric in 37 patients, transduodenal in 13 patients, while 7 patients had both (2 multigated and 5 sequential). Clinical success (resolution of PFCs) was achieved in 15 patients (71.4\%) in the LAMSs alone group, and 21 patients (58.3\%) with LAMSs plus DPSs $(\mathrm{P}=0.32)$. The median stent duration was 7 weeks in LAMSs group and 8 weeks in LAMSs + DPSs (Fig. 3, Table 2).

In patients with LAMSs alone, $6(28.6 \%)$ patients had adverse events: 1 (16.6\%) bleeding, 1 (16.6\%) stent migration, and $4(66.6 \%)$ stent obstruction $(\mathrm{P}=0.43)$. In those with LAMSs

Table 1 Demographic data and characteristics of PFCs

\begin{tabular}{|c|c|c|c|}
\hline Parameter & LAMSs alone $(\mathrm{n}=21)$ & LAMSs+DPSs $(n=36)$ & P-value \\
\hline \multicolumn{4}{|l|}{ Age (years) } \\
\hline Mean & 48.95 & 46.61 & 0.59 \\
\hline \multicolumn{4}{|l|}{ Sex } \\
\hline Female & $10(47.6 \%)$ & $13(36.1 \%)$ & 0.39 \\
\hline Male & $11(52.4 \%)$ & $23(63.9 \%)$ & \\
\hline \multicolumn{4}{|l|}{ Pancreatitis etiology } \\
\hline Alcohol & $5(23.8 \%)$ & $6(16.7 \%)$ & 0.78 \\
\hline Gallstones & $8(38.1 \%)$ & $14(38.9 \%)$ & \\
\hline Others & $8(38.1 \%)$ & $16(44.4 \%)$ & \\
\hline \multicolumn{4}{|l|}{ PPI use } \\
\hline No & $12(57.1 \%)$ & $23(63.9 \%)$ & 0.61 \\
\hline Yes & $9(42.9 \%)$ & $13(36.1 \%)$ & \\
\hline \multicolumn{4}{|l|}{ Drainage site } \\
\hline Both & $0(0.0 \%)$ & $7(19.4 \%)$ & 0.09 \\
\hline Transduodenal & $5(23.8 \%)$ & $8(22.2 \%)$ & \\
\hline Transgastric & $16(76.2 \%)$ & $21(58.3 \%)$ & \\
\hline \multicolumn{4}{|l|}{ PFCs type } \\
\hline Pancreatic pseudocyst & $7(33.3 \%)$ & $7(19.4 \%)$ & 0.24 \\
\hline Walled-off pancreatic necrosis & $14(66.7 \%)$ & $29(80.6 \%)$ & \\
\hline \multicolumn{4}{|l|}{ PFCs size (mm) } \\
\hline$<50$ & $1(4.8 \%)$ & $2(5.6 \%)$ & 0.25 \\
\hline $51-100$ & $14(66.7 \%)$ & $16(44.4 \%)$ & \\
\hline$>101$ & $6(28.6 \%)$ & $18(50.0 \%)$ & \\
\hline \multicolumn{4}{|l|}{ Nutrition type } \\
\hline Parenteral nutrition & $0(0.0 \%)$ & $1(2.8 \%)$ & 0.34 \\
\hline Oral & $12(57.1 \%)$ & $14(38.9 \%)$ & \\
\hline PEG-J & $9(42.9 \%)$ & $21(58.3 \%)$ & \\
\hline The average number of necrosectomies & 1.14 & 1.56 & 0.39 \\
\hline \multicolumn{4}{|l|}{ Percutaneous drainage } \\
\hline No & $21(100.0 \%)$ & $30(83.3 \%)$ & 0.04 \\
\hline Yes & $0(0.0 \%)$ & $6(16.7 \%)$ & \\
\hline Median stent duration (weeks) & 7.38 & 7.86 & 0.35 \\
\hline
\end{tabular}

LAMSs, lumen-apposing metal stents; DPSs, double-pigtail plastic stents; PFCs, pancreatic fluid collections; PPI, proton pump inhibitor; PEG-J, percutaneous endoscopic transgastric jejunostomy 


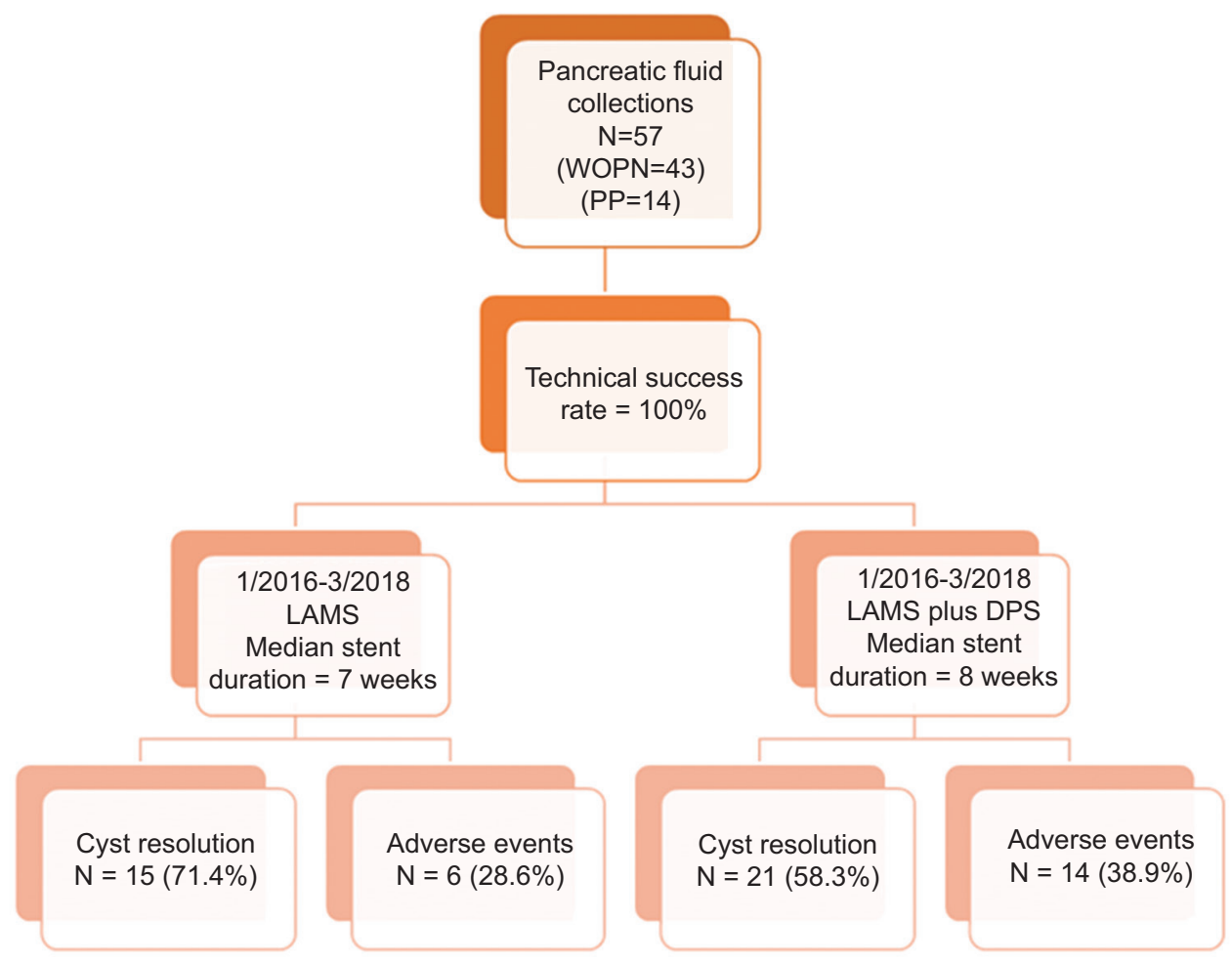

Figure 3 Flowchart of patients with pancreatic fluid collections, showing outcomes

WOPN, walled-off pancreatic necrosis; PP, pancreatic pseudocyst; LAMS, lumen-apposing metal stent; DPS, double-pigtail plastic stent

plus DPSs, $14(38.9 \%)$ patients had adverse events: $2(14.2 \%)$ bleeding, $6(42.8 \%)$ stent migration, and $6(42.8 \%)$ stent obstruction $(\mathrm{P}=0.43)$ (Table 3$)$. The most common adverse event was stent obstruction, followed by stent migration and bleeding (Fig. 4). Most of the complications occurred postprocedure, in the first 14 days: 3 (50\%) patients in the LAMSs alone and $9(64.2 \%)$ patients in the LAMSs plus DPSs $(\mathrm{P}=0.28)$. Infection was not reported among any of the study participants (Table 3).

\section{Discussion}

Patients with non-resolving symptomatic PFCs may have a prolonged hospital course with high morbidity and mortality. EUS-guided drainage has become the first method of drainage for symptomatic PFCs, with technical and clinical success rates more than $90 \%$ and $75 \%$, respectively [15-18]. The use of LAMSs in EUS-guided drainage of PFCs has been reported to be associated with a higher risk of adverse events compared with other types of stent, and this risk can be reduced by placing a coaxial DPSs with the LAMSs $[8,19,20]$. Currently, there are no predefined selection criteria for using the LAMSs alone or LAMSs plus DPSs during the drainage of PFCs. In this study, we found that anchoring coaxial DPSs to LAMSs was not associated with a lower rate of adverse events or a higher rate of cyst resolution in EUS-guided drainage of PFCs. Since the introduction of LAMSs, drainage of PFCs has become much
Table 2 Cyst resolution and adverse events

\begin{tabular}{lccc}
\hline Parameter & $\begin{array}{c}\text { LAMSs alone } \\
(\mathrm{n}=21)\end{array}$ & $\begin{array}{c}\text { LAMSs+DPSs } \\
(\mathrm{n}=36)\end{array}$ & P-value \\
& & & \\
\hline $\begin{array}{l}\text { Cyst resolution } \\
(\text { No })\end{array}$ & $6(28.6 \%)$ & $15(41.7 \%)$ & 0.32 \\
$($ Yes $)$ & $15(71.4 \%)$ & $21(58.3 \%)$ & \\
Adverse events & & & \\
$($ No) & $15(71.4 \%)$ & $22(61.1 \%)$ & 0.43 \\
$($ Yes $)$ & $6(28.6 \%)$ & $14(38.9 \%)$ & \\
\hline
\end{tabular}

LAMSs, lumen-apposing metal stents; DPSs, double-pigtail plastic stents

Table 3 Rate and timing of adverse events in patients with LAMSs alone vs. LAMSs plus DPSs

\begin{tabular}{llll}
\hline Parameter & $\begin{array}{l}\text { LAMSs } \\
(\mathrm{n}=6)\end{array}$ & $\begin{array}{l}\text { LAMSs + DPSs } \\
(\mathrm{n}=14)\end{array}$ & P-value \\
\hline $\begin{array}{lll}\text { Adverse event rate } \\
\text { Stent obstruction }\end{array}$ & $\begin{array}{l}6(28.6 \%) \\
\text { Stent migration }\end{array}$ & $14(38.9 \%)$ & 0.43 \\
Bleeding & $1(16.6 \%)$ & $6(42.8 \%)$ & \\
Timing of adverse events & $1(16.6 \%)$ & $2(14.2 \%)$ & 0.28 \\
Intra-procedure & $1(16.6 \%)$ & 0 & \\
Post-procedure & $3(50 \%)$ & $9(64.2 \%)$ & \\
Late & $2(33.3 \%)$ & $5(35.7 \%)$ & \\
\hline LAMSs, lumen-apposing metal stents; DPSs, double-pigtail plastic stents
\end{tabular}

easier, because a LAMS's internal diameter is large enough for the endoscope to pass through, allowing endoscopic 


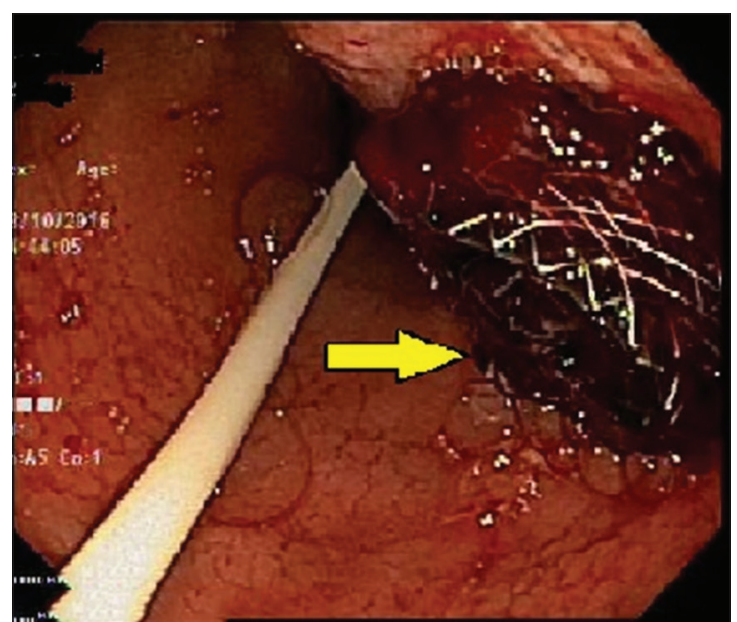

Figure 4 Endoscopic image showing the lumen-opposing metal stent with the yellow arrow pointing to the bleeding around it

necrosectomy with a success rate of up to $90 \%[7,11,15]$. LAMSs are used mainly in patients with WOPN where necrosectomy is required, but their role in the drainage of PP is still not entirely clear, as a PP can be effectively drained by DPSs [21]. Endoscopic drainage using DPSs is very effective in PP, with a clinical success rate of $88-98 \%$, but in the case of WOPN the drainage is not optimal, with a clinical success rate of $63-70 \%$ [21-23]. According to Siddiqui et al in a recent report, the mean number of procedures needed for resolution of WOPN at 6-months follow up was lower in patients with LAMSs compared with fully covered self-expandable metal stents (FCSEMSs) and DPSs 2.2 vs. 3 vs. 3.6, respectively; $\mathrm{P}=0.04$ ) [8]. Also, rate of resolution of WOPN at 6-month follow up was lower in patients with DPSs compared with FCSEMSs and LAMSs ( $81 \%$ vs. $95 \%$ vs. $90 \%$; $\mathrm{P}=0.001$ ) [8]. FCSEMSs have a larger diameter lumen compared with DPSs, and this can allow more efficient drainage with less risk of stent occlusion and superimposed infection. Therefore, they have been used for the drainage of PFCs [24,25]. However, the main disadvantage of FCSEMSs was the high migration rate, leading to inefficient drainage and leak [26]. The technical success rate of FCSEMSs is $78-100 \%$ and the clinical success rate is above $80 \%[8,18,22,23,27]$. According to Sharaiha et al [15], 16 patients with DPSs were about 2.9 times more likely to have adverse events than those patients with FCSEMSs.

LAMSs are designed with a bi-flanged shape that allows for tissue apposition and minimizes the risk of stent migration. They have a wide diameter lumen that provides a non-compressible fistulous tract between the gut lumen and the pancreatic fluid cavity. In addition, it can provide the channel for endoscopic necrosectomy and the need for repeated endoscopies. The clinical and technical success rates of LAMSs have been reported to be $93-100 \%$ and 89 $100 \%$, respectively $[7,11,23,25,28-30]$. LAMSs have become the stent of choice for endoscopic drainage of PFCs by many gastroenterologists, because of their easy deployment and direct debridement access. According to Siddiqui et al [7], in a multicenter retrospective study that assessed EUS-guided

\section{Summary Box}

\section{What is already known:}

- Lumen-apposing metal stents (LAMSs) are the stents of choice for the endoscopic drainage of pancreatic fluid collections (PFCs)

- Inserting a double-pigtail plastic stent (DPS) through the LAMS has been proposed to be safer than LAMS alone and can help decrease adverse events

\section{What the new findings are:}

- Anchoring coaxial DPSs to LAMSs was not associated with a higher rate of fluid resolution during endoscopic ultrasound-guided drainage of PFCs

- There was no significant difference in adverse events between LAMS and LAMS plus DPS

drainage of symptomatic PFCs in 82 patients, the adverse event rate was $9.8 \%$. The adverse events among these patients were 2 maldeployed stents, 6 episodes of bleeding, 5 episodes of PFC infection, and 4 stent occlusions. Also, per Siddiqui et al [8] compared FCSEMs vs. LAMSs vs. DPSs for the drainage of WOPN. The results showed that LAMSs were more likely to be associated with early adverse events compared with FCSEMSs $(\mathrm{P}=0.02)$ and bleeding was the most frequently reported complication. Recently, a pilot study by Aburajab et al [31] reported that EUS-guided drainage of PP via LAMSs is associated with higher rates of infection and nonresolution, but these adverse events can be minimized by placing DPSs across the LAMSs.

In our study, bleeding was not the most frequent event. It was reported in only one patient in the LAMSs group, during the procedure, and in 2 patients with LAMSs plus DPSs, with 1 episode occurring post-procedure and the other late. The most reported adverse event in both groups was stent obstruction by necrotic debris. There was no significant difference between LAMSs and LAMSs plus DPSs in terms of adverse events, while the median stent duration was 7 weeks in the LAMSs group and 8 weeks in the LAMSs plus DPSs group.

The limitations of this study are its retrospective design with a small sample size, a single-center experience, selection bias, no well-known selection criteria for the LAMSs alone or LAMSs plus DPSs approaches (the endoscopist made the decision on which approach to use), and generalizability limitations.

In conclusion, this study showed that anchoring coaxial DPSs to LAMSs was not associated with a lower rate of adverse events or a higher rate of cyst resolution in EUS-guided drainage of PFCs. Further studies with larger sample sizes are needed to validate the results. 


\section{References}

1. Peery AF, Crockett SD, Barritt AS, et al. Burden of gastrointestinal, liver, and pancreatic diseases in the United States. Gastroenterology 2015;149:1731-1741.

2. Yadav D, Whitcomb DC. The role of alcohol and smoking in pancreatitis. Nat Rev Gastroenterol Hepatol 2010;7:131-145.

3. Johnson CD, Abu-Hilal M. Persistent organ failure during the first week as a marker of fatal outcome in acute pancreatitis. Gut 2004;53:1340-1344.

4. Banks PA, Bollen TL, Dervenis C, et al; Acute Pancreatitis Classification Working Group. Classification of acute pancreatitis-2012: revision of the Atlanta classification and definitions by international consensus. Gut 2013;62:102-111.

5. Varadarajulu S, Christein JD, Tamhane A, Drelichman ER, Wilcox CM. Prospective randomized trial comparing EUS and EGD for transmural drainage of pancreatic pseudocysts (with videos). Gastrointest Endosc 2008;68:1102-1111.

6. Singhal S, Rotman SR, Gaidhane M, Kahaleh M. Pancreatic fluid collection drainage by endoscopic ultrasound: an update. Clin Endos 2013;46:506-514.

7. Siddiqui AA, Adler DG, Nieto J, et al. EUS-guided drainage of peripancreatic fluid collections and necrosis by using a novel lumen-apposing stent: a large retrospective, multicenter U.S. experience (with videos). Gastrointest Endosc 2016;83:699-707.

8. Siddiqui AA, Kowalski TE, Loren DE, et al. Fully covered selfexpanding metal stents vs. lumen-apposing fully covered selfexpanding metal stent vs. plastic stents for endoscopic drainage of pancreatic walled-off necrosis: clinical outcomes and success. Gastrointest Endosc 2017;85:758-765.

9. Chen, Yen-I. Barkun AN2, Adam V, et al. Cost-effectiveness analysis comparing lumen apposing metal stents with plastic stents in the management of pancreatic walled-off necrosis. Gastrointest Endosc 2018;88:267-276.e1.

10. Puga M, Consiglieri CF, Busquets J, et al. Safety of lumenapposing stent with or without coaxial plastic stent for endoscopic ultrasound-guided drainage of pancreatic fluid collections: a retrospective study. Endoscopy 2018;50:1022-1026.

11. Sharaiha RZ, Tyberg A, Khashab MA, et al. Endoscopic therapy with lumen-apposing metal stents is safe and effective for patients with pancreatic walled-off necrosis. Clin Gastroenterol Hepatol 2016;14:1797-1803.

12. Lang GD, Fritz $C$, Bhat $T$, et al. EUS-guided drainage of peripancreatic fluid collections with lumen-apposing metal stents and plastic double-pigtail stents: comparison of efficacy and adverse event rates. Gastrointest Endosc 2018;87:150-157.

13. Working Group IAP/APA Acute Pancreatitis Guidelines. IAP/ APA evidence-based guidelines for the management of acute pancreatitis. Pancreatology 2013;13:e1-e15.

14. Cotton PB, Eisen GM, Aabakken L, et al. A lexicon for endoscopic adverse events: report of an ASGE workshop. Gastrointest Endosc 2010;71:446-454.

15. Sharaiha RZ, DeFilippis EM, Kedia P, et al. Metal versus plastic for pancreatic pseudocyst drainage: clinical outcomes and success. Gastrointest Endosc 2015;82:822-827.

16. Sadik R, Kalaitzakis E, Thune A, Hansen J, Jönson C. EUS-guided drainage is more successful in pancreatic pseudocysts compared with abscesses. World J Gastroenterol 2011;17:499-505.
17. Gluck M, Ross A, Irani S, et al. Endoscopic and percutaneous drainage of symptomatic walled-off pancreatic necrosis reduces hospital stay and radiographic resources. Clin Gastroenterol Hepatol 2010;8:1083-1088.

18. Varadarajulu S, Wilcox CM, Latif S, Phadnis M, Christein JD. Management of pancreatic fluid collections: a changing of the guard from surgery to endoscopy. Am Surg 2011;77:1650-1655.

19. Bang JY, Hasan M, Navaneethan U, Hawes R, Varadarajulu S. Lumenapposing metal stents (LAMS) for pancreatic fluid collection (PFC) drainage: may not be business as usual. Gut 2017;66:2054-2056.

20. Gornals JB, Consiglieri CF, Busquets J, et al. Endoscopic necrosectomy of walled-off pancreatic necrosis using a lumenapposing metal stent and irrigation technique. Surg Endosc 2016;30:2592-2602.

21. Bang JY, Hawes R, Bartolucci A, Varadarajulu S. Efficacy of metal and plastic stents for transmural drainage of pancreatic fluid collections: a systematic review. Dig Endosc 2015;27:486-498.

22. Bang JY, Varadarajulu S. Metal versus plastic stent for transmural drainage of pancreatic fluid collections. Clin Endosc 2013;46:500-502.

23. Vazquez-Sequeiros E, Baron TH, Pérez-Miranda M, et al; Spanish Group for FCSEMS in Pancreas Collections. Evaluation of the short- and long-term effectiveness and safety of fully covered selfexpandable metal stents for drainage of pancreatic fluid collections: results of a Spanish nationwide registry. Gastrointest Endosc 2016;84:450-457.

24. Talreja JP, Shami VM, Ku J, Morris TD, Ellen K, Kahaleh M. Transenteric drainage of pancreatic-fluid collections with fully covered self-expanding metallic stents (with video). Gastrointest Endosc 2008;68:1199-1203.

25. Shah RJ, Shah JN, Waxman I, et al. Safety and efficacy of endoscopic ultrasound-guided drainage of pancreatic fluid collections with lumen-apposing covered self-expanding metal stents. Clin Gastroenterol Hepatol 2015;13:747-752.

26. Baron TH, Harewood GC, Morgan DE, Yates MR. Outcome differences after endoscopic drainage of pancreatic necrosis, acute pancreatic pseudocysts, and chronic pancreatic pseudocysts. Gastrointest Endosc 2002;56:7-17.

27. Singhal S, Rotman SR, Gaidhane M, Kahaleh M. Pancreatic fluid collection drainage by endoscopic ultrasound: an update. Clin Endosc 2013;46:506-514.

28. Rinninella E, Kunda R, Dollhopf M, et al. EUS-guided drainage of pancreatic fluid collections using a novel lumen-apposing metal stent on an electrocautery-enhanced delivery system: a large retrospective study (with video). Gastrointest Endosc 2015;82:1039-1046.

29. Itoi T, Binmoeller KF, Shah J, et al. Clinical evaluation of a novel lumen-apposing metal stent for endosonography-guided pancreatic pseudocyst and gallbladder drainage (with videos). Gastrointest Endosc 2012;75:870-876.

30. Gornals JB, De la Serna-Higuera C, Sánchez-Yague A, Loras C, Sánchez-Cantos AM, Pérez-Miranda M. Endosonography-guided drainage of pancreatic fluid collections with a novel lumenapposing stent. Surg Endosc 2013;27:1428-1434.

31. Aburajab M, Smith Z, Khan A, Dua K. Safety and efficacy of lumen-apposing metal stents with and without simultaneous double-pigtail plastic stents for draining pancreatic pseudocyst. Gastrointest Endosc 2018;87:1248-1255. 